\title{
Culture costs lives
}

\section{Vaccine: The Controversial Story of \\ Medicine's Greatest Lifesaver \\ by Arthur Allen \\ Norton: 2007.512 pp. £17.99/\$27.95}

\section{Michael B. A. Oldstone}

Vaccines, like antibiotics and improvements to sanitation and water quality, have greatly affected human health. Vaccines play a commanding role in controlling such formerly devastating plagues as smallpox, yellow fever, measles, poliomyelitis, tetanus and diphtheria. The first vaccine to reduce the risk of developing liver cancer (hepatitis B) is now a reality. Nevertheless, some continue to regard vaccination as controversial, as Arthur Allen describes in his book on the evolution of vaccines, associated fears and dissatisfactions as well as related politics and players.

Allen is an investigative reporter who learned, just before his son was to receive a whooping-cough vaccination, that a new, 'safe' vaccine was replacing an older 'less safe' one. He then began researching the controversy over vaccines and the question of mandatory vaccination for those attending state-funded schools in the United States. He analyses why about $25 \%$ of US parents are reluctant to have children vaccinated, the social history of vaccination and the reasons for public ambivalence. Allen also deals with use of the Internet to obtain medical information that is frequently subjective, emotional and erroneous.

Routine immunizations to prevent crippling polio or measles are carried out in developed countries, such as the United States. The rationale for such mass vaccination is that if sufficient numbers of a population are vaccinated, the incidence of disease will be low or nonexistent due to herd immunity. As Allen notes, those in the basic sciences or medicine consider such issues objectively - that is, is the benefit of preventing disease greater than the risk to those vaccinated? Other opinions often reflect the political divide in the United States between those who believe in a free market and individual choice, and those who favour collective responsibility and government financing of programmes to protect the health of all children.

Allen's early chapters on the origins of vaccination are adequate, although his description of medical practices in the eighteenth and nineteenth centuries seems excessive, considering that the abysmal usage of purges, poisons and bleeding before normal physiology and events causing disease were understood. That bacteria or their toxins cause disease has only been known for just over 160 years, viruses were first isolated

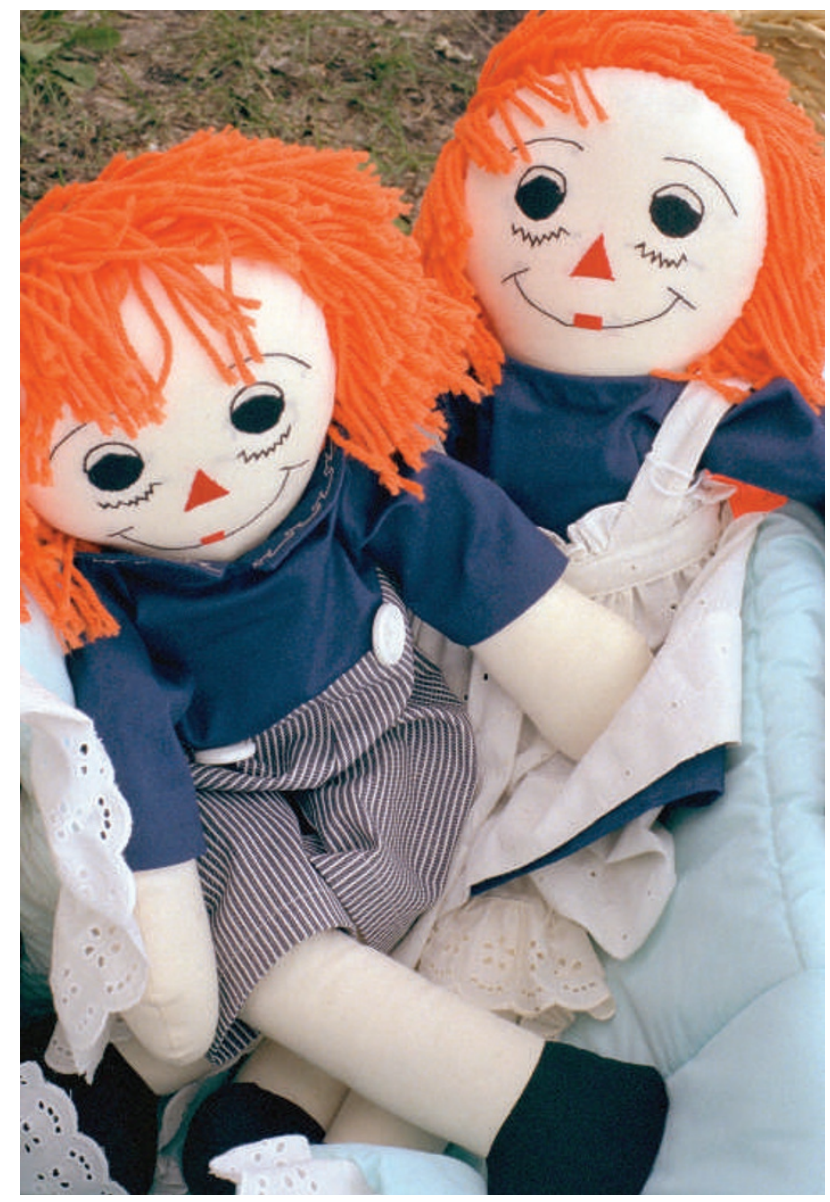

Raggedy Ann became the symbol of a vaccine-damaged child.

little more than 100 years ago and the emergence of initial concepts about immunology and allergy followed. The history of Edward Jenner and the first vaccine (smallpox) along with the difficulty of its contamination with diverse infectious agents are well detailed. Interestingly, the religious objection dating back to Jenner's time in the late 1700s that man must not, by using vaccines, interfere with God's will has not abated. Similar arguments, and others, are raised today against using the vaccine for the sexually transmitted human papilloma virus - to prevent cervical cancer - in the United States and the polio vaccine in Nigeria.

Some factual errors mar certain chapters, and others are difficult to follow. For example, the chapter on measles also covers diphtheria, pertussis (whooping cough), tetanus, SV40 (simian virus), HIV/AIDS, varicella (chicken pox), hepatitis B, Streptococcus, respiratory syncytial virus, cowpox and the flu epidemic of 1919, a mix sufficient to disturb any reductionist.

Nevertheless, the book has several gems that will intrigue readers. One is the origin Congressmen Dan Burton (Republican, Indiana) and Curt Weldon (Republican, Pennsylvania), their attack on the National Immunization Program and their work with antivaccination groups. They argue that legislatures, as representatives of the people, and not scientists or public-health workers should decide which vaccines to administer to children. The proposition leaves us, to quote Allen, "....punched in the nose by a congressional bully. It does not matter that they were smart kids (scientists, public health officials), he (the bully) still controls the playground." In the words of the late Democratic senator for New York, Daniel Patrick Moynihan: "Every man is entitled to his own opinion, but not to his own facts."

What becomes clear throughout this book is that, when facts tangle with culture, culture often wins. For those interested in the politics and debate of compulsory vaccination, and the personalities involved in all sides of the fight, Vaccine is a good read.

Michael B. A. Oldstone is professor and head of the Viral-Immunobiology Laboratory in the Molecular and Integrative Neurosciences Department, The Scripps Research Institute, 10550 North Torrey Pines Road, La Jolla,

California 92037, USA. 